\title{
Physico-chemicals and catalytic properties of manganese-promoted vanadium phosphate (VPO) catalyst
}

\begin{abstract}
The addition of $1 \% \mathrm{Mn}$ promoter to vanadium phosphate catalyst led to doubling of the specific surface area from 20.3 (unpromoted) to $39.4 \mathrm{~m} 2 \mathrm{~g}-1$. The XRD pattern of the Mnpromoted catalyst gave only the characteristics of the (VO)2P2O7 phase, indicating that the Mn was incorporated into the crystal lattice of the catalyst. The Mn-promoted catalyst was also twice as active in removing the total amount of oxygen. However, since the only oxygen species related to V4+ being removed and no oxygen species associated with V5+ was observed, the n-butane conversion was not much improved as compared to the unpromoted counterpart. A necessary amount and distribution of the V5+ phase in a well crystalline V4+ phase is essential in order to enhance the catalytic performance in the mild oxidation of nbutane to maleic anhydride.
\end{abstract}

Keyword: Vanadium phosphate, Mn, butane oxidation, maleic anhydride 